\title{
Desempenho da modelagem cinemática do escoamento superficial para chuvas intermitentes em solos com cobertura morta
}

\author{
João R. C. B. Abrantes ${ }^{1}$, João L. M. P. de Lima² \& Abelardo A. A. Montenegro ${ }^{3}$
}

\section{Palavras-chave:}

simulador de laboratório

onda cinemática

conservação do solo

\begin{abstract}
R E S U M O
A cobertura morta é uma prática de manejo imprescindível para o controle do escoamento superficial. Condições antecedentes de umidade do solo, padrões de precipitação e cobertura do solo têm relevância no desprendimento, no transporte das partículas sólidas e na geração de escoamento superficial. Este estudo objetivou avaliar o desempenho do modelo da onda cinemática no escoamento superficial oriundo de eventos de chuvas intermitentes de múltiplos picos considerando-se diferentes densidades de cobertura. Ensaios de laboratório foram realizados em um canal de terra e com um simulador de chuva adotando-se três cenários de cobertura do solo: i) Solo nu; ii) Cobertura morta, com densidade de $2 \mathrm{t} \mathrm{ha}^{-1} \mathrm{e}$ iii) Cobertura morta com densidade de $4 \mathrm{t} \mathrm{ha}^{-1}$. Os experimentos mostram, de forma bem clara, que a cobertura morta afeta fortemente o escoamento superficial. A intermitência e as características das chuvas sequenciais também influenciaram tal processo. O modelo da onda cinemática previu eficientemente o escoamento superficial apresentando coeficiente de Nash-Sutcliffe superior a 0,75 e coeficiente de determinação maior que 0,90, para todas as condições de cobertura, quando a umidade inicial do solo era elevada.
\end{abstract}

\section{Key words:}

laboratory simulator

kinematic wave

soil conservation

\section{Performance of kinematic modelling of surface runoff for intermittent rainfall on soils covered with mulch}

\begin{abstract}
A B S T R A C T
Mulching is an important management practice for overland flow control. Antecedent soil moisture conditions, rainfall patterns, and soil cover play an important role in the detachment and transport of soil particles and runoff generation. This study aimed to investigate the performance of kinetic wave model in simulating overland flow from multiple step intermittent rainfall events, considering distinct mulch densities. Laboratory experiments were conducted using a soil flume and rainfall simulator adopting three soil cover scenarios: i) Bare soil; ii) Low mulch cover density of $2 \mathrm{t} \mathrm{ha}^{-1}$; and iii) High mulch cover density of $4 \mathrm{tha}^{-1}$. The laboratory experiments clearly show that mulch strongly affects surface runoff. Intermittency and characteristics of sequential rainfall events also influenced such process. The kinematic wave model predicted efficiently surface runoff, presenting Nash-Sutcliffe coefficients higher than 0,75 and determination coefficients higher than 0,90 , for all cover conditions, when initial soil moisture was high.
\end{abstract}

Protocolo 408.13 - 16/12/2013 • Aprovado em 03/10/2014 • Publicado em 01/02/2015

${ }^{1}$ IMAR/MARE - DEC/FCTUC. Coimbra, Portugal. E-mail: jrcbrito@msn.com

${ }^{2}$ IMAR/MARE - DEC/FCTUC. Coimbra, Portugal. E-mail: plima@dec.uc.pt

${ }^{3}$ DTR/UFRPE. Recife, PE. E-mail: abelardo.montenegro@yahoo.com.br (Autor correspondente) 


\section{INTRODUÇÃo}

Em regiões semiáridas a gestão das práticas de conservação do solo é fundamental para o aumento da eficiência no uso da água. As técnicas conservacionistas, dentre elas a cobertura morta, se constituem em alternativas eficazes e de baixo custo para a redução do escoamento superficial, aumento da infiltração e da umidade do solo e incremento da produção agrícola (Santos et al., 2011).

Chen et al. (2007) estudaram o efeito de diferentes condições de superfície na umidade do solo e geração de escoamento superficial e verificaram que a vegetação formada por gramíneas e plantas herbáceas foi efetiva na conservação da umidade e na redução do escoamento superficial. Pan \& Shangguan (2006) também observaram que a cobertura com grama originou redução da velocidade do escoamento superficial.

Montenegro et al. (2013) por sua vez, constataram que a cobertura morta de palha com densidades de 2 e $4 \mathrm{t} \mathrm{ha}^{-1}$, foi eficiente na redução do escoamento superficial e da temperatura do solo, além de promover maior conteúdo de água no solo. Shen et al. (2012) avaliaram o efeito de diferentes taxas de cobertura morta $\left(0,6\right.$ e $\left.12 \mathrm{t} \mathrm{ha}^{-1}\right)$ no solo verificando que esta influenciou significativamente o conteúdo de água do solo, reduziu o escoamento superficial e promoveu a produção agrícola. Santos et al. (2010) também observaram um efeito positivo e significativamente superior no rendimento de feijão com a adoção de tratamentos conservacionistas (e.g. aplicação de cobertura morta com barramentos de pedra).

Na natureza se sucedem, usualmente, chuvas intermitentes que ainda não foram tão amplamente estudadas como as chuvas isoladas, em termos de experimentação e modelagem. Tais chuvas produzem efeitos diferenciados no balanço entre escoamento superficial e infiltração, a depender da distribuição temporal de seus picos. Deng et al. (2008) desenvolveram simulações de laboratório e numéricas para investigar o formato de hidrogramas para escoamento e sedimentos, produzidos por chuvas de intensidade variável. Römkens et al. (2001) verificaram que uma sequência de eventos de chuva de intensidade decrescente causa perdas de solo mais elevadas que uma sequência simétrica com valores crescentes. Ran et al. (2012) observaram que os padrões hidrológicos das chuvas, particularmente as intensidades e durações dos picos, são de elevada relevância afetando o escoamento superficial e o transporte de sedimentos.

A investigação em laboratório através de simuladores de chuva, é de grande valia para o entendimento dos processos hidrológicos decorrentes de chuvas naturais, permitindo o detalhamento e a reprodução de forma controlada de eventos complexos. Assim, a simulação de chuvas proporciona o controle das características temporais e espaciais da precipitação, tal como a obtenção rápida de dados. Tais fatores garantem algumas vantagens na utilização de simuladores de chuva em estudos relacionados com o escoamento e à infiltração, tanto em laboratório como in situ (Cerdà et al., 1997; Lima et al., 2002; Pérez-Latorre et al., 2010).

Os modelos numéricos são sumamente importantes para avaliação de cenários e para a gestão ambiental constituindo-se em instrumentos essenciais de apoio à tomada de decisão. Em escoamentos pouco profundos o modelo da onda cinemática tem sido utilizado por muitos investigadores dado à sua aproximação ao modelo dinâmico (Singh, 1996; Lima \& Singh, 2002; Deng et al., 2008). A comprovação experimental tem sido bastante investigada (Molen et al., 1995). Dentre as aplicações recentes do modelo da onda cinemática pode-se citar o estudo desenvolvido por Giesen et al. (2011) avaliando a relevância da escala espacial na geração de escoamento superficial em encostas utilizadas para a agricultura, porém o desempenho de tal modelo em condições de chuva intermitente e elevada rugosidade superficial, como aquelas cobertas de palha, ainda não é adequadamente estabelecido.

De posse do acima exposto objetiva-se, neste estudo, avaliar o desempenho do modelo da onda cinemática na simulação do escoamento superficial na presença de diferentes densidades de cobertura morta e para diferentes sequências de chuvas simuladas.

\section{Material e Métodos}

Os experimentos laboratoriais foram realizados na instalação apresentada na Figura 1A, constituída de um simulador de chuva do tipo aspersor e um canal de terra retangular com 3,00 $\mathrm{m}$ de comprimento, $0,30 \mathrm{~m}$ de largura e $0,12 \mathrm{~m}$ de profundidade, fixado a uma inclinação de $10 \%$. (Lima et al., 2003; Montenegro et al., 2013).

O simulador de chuva é composto por um sistema aspersor constituído por um nebulizador de cone preenchido $(3 / 8 \mathrm{HH}$ FullJet da Spraying Systems Co.) orientado para baixo com um ângulo de aspersão de $90^{\circ}$ e um orifício de aspersão de $4,8 \mathrm{~mm}$ posicionado a $2,20 \mathrm{~m}$ acima do centro geométrico do canal de terra. O sistema aspersor é alimentado com pressão constante de 0,14 MPa através de um sistema de bombeamento com um reservatório de nível constante, uma bomba submersível, um tanque e um sensor de pressão.

O simulador de chuva está fixado a uma estrutura de suporte motorizada permitindo um movimento controlado na direção transversal ao comprimento do canal de terra e nos dois sentidos. Desta, foram definidas três posições do aspersor que corresponderam a três intensidades de precipitação distintas sobre o canal de terra apresentadas na Figura $2 \mathrm{~A} \mathrm{e}$ na Tabela 1. Apesar dos baixos coeficientes de uniformidade, o sistema representa adequadamente uma situação natural

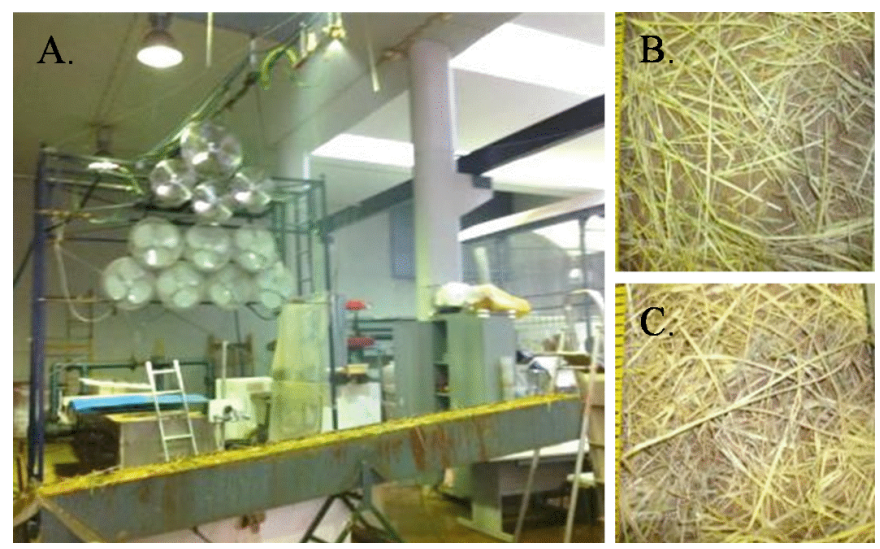

Figura 1. Fotografias das experiências laboratoriais: Vista geral da instalação laboratorial com o simulador de chuva e canal de terra (A), Superfície do solo com $2 \mathrm{t} \mathrm{ha}^{-1}$ de cobertura morta (B), Superfície do solo com $4 \mathrm{t} \mathrm{ha}^{-1}$ de cobertura morta $(C)$. Note-se que o sistema de geração de vento (Figura 1A) não foi utilizado no presente estudo 

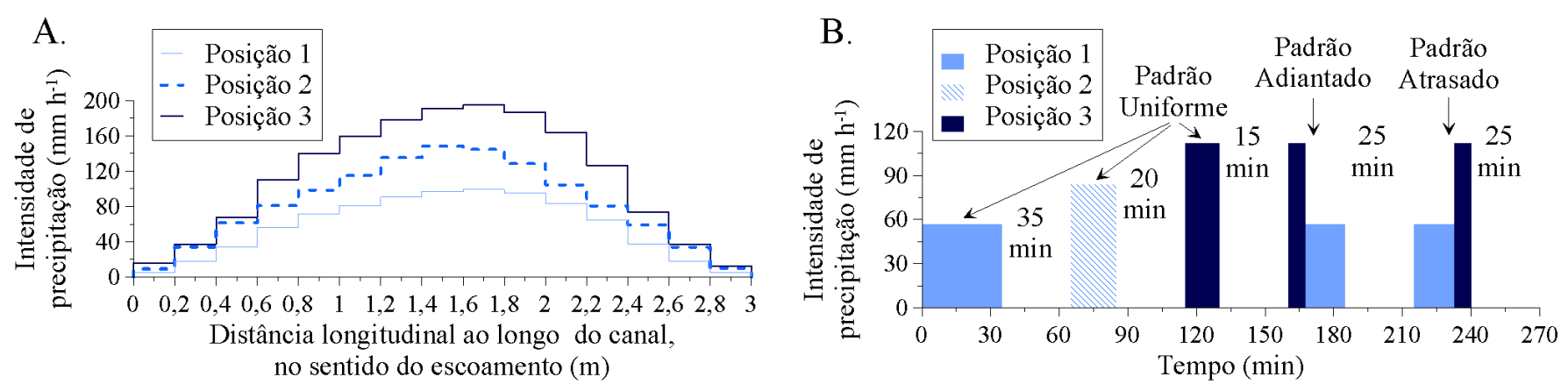

Figura 2. Eventos de precipitação simulados em cada experimento: Distribuição espacial da intensidade de precipitação a nível do canal de terra (intensidades médias para a largura do canal), para as três posições do nebulizador (A), Sequência temporal dos 5 eventos de precipitação intercalados de períodos secos (B)

Tabela 1. Características da precipitação simulada pelo nebulizador, a nível do canal de terra para as três posições definidas

\begin{tabular}{lccc}
\hline & \multicolumn{4}{c}{ Posição do canal } \\
\cline { 2 - 4 } & $\mathbf{1}$ & $\mathbf{2}$ & $\mathbf{3}$ \\
Distância horizontal até 0 aspersor $(\mathrm{m})$ & 0 & 0,45 & 0,90 \\
Intensidade média de precipitação $\left(\mathrm{mm} \mathrm{h}^{1}\right)$ & 112 & 84 & 57 \\
Intensidade máxima de precipitação $\left(\mathrm{mm} \mathrm{h}^{1}\right)$ & 243 & 176 & 118 \\
Intensidade mínima de precipitação $\left(\mathrm{mm} \mathrm{h}^{1}\right)$ & 11 & 7 & 2 \\
Coeficiente de uniformidade $(\%)^{1}$ & 47,1 & 43,7 & 37,3 \\
Diâmetro médio das gotas $(\mathrm{mm})^{2}$ & 0,70 & 0,77 & 0,77 \\
Velocidade média das gotas $\left(\mathrm{m} \mathrm{s}^{-1}\right)$ & 2,59 & 2,58 & 2,80 \\
\hline
\end{tabular}

${ }_{1}^{1}$ Os coeficientes de uniformidade foram calculados de acordo com Christiansen (1942)

${ }^{2} 0$ diâmetro médio e a velocidade média das gotas foram medidos usando-se um disdrômetro a laser (ThiesClima $\left.{ }^{\circledR}\right)$

em que a precipitação não é espacialmente uniforme na área de drenagem.

Utilizando as três intensidades de precipitação (Figura 2A) definiu-se o procedimento experimental esquematizado na Figura $2 \mathrm{~B}$ incluiundo a simulação de uma sequência de cinco eventos de precipitação intercalados por períodos secos de $30 \mathrm{~min}$ (i.e. períodos sem chuva). Os cinco eventos de precipitação apresentaram intensidades, durações e padrões temporais e espaciais diferentes, porém cada um produziu aproximadamente o mesmo volume total de precipitação. Durante os períodos sem chuva foram utilizadas lâmpadas de infravermelho para promover a evaporação da água contida no solo e na cobertura morta (i.e. palha).

O solo utilizado nos experimentos foi recolhido nas margens do Rio Mondego (Coimbra, Portugal) e classificado com o de textura franco-arenosa ( $84 \%$ de areia, $9 \%$ de limo e $7 \%$ de argila). O solo foi secado ao ar, peneirado em malha de $2 \mathrm{~mm}$, espalhado uniformemente no canal de terra, nivelado e compactado até se obter uma camada uniforme de solo, com espessura de $0,10 \mathrm{~m}$ e densidade (natural) de 1,340 $\mathrm{kg} \mathrm{dm}^{-3}$. O solo apresentou condutividade hidráulica de 5,7 $\pm 1,8 \times 10^{-5} \mathrm{~m} \mathrm{~s}^{-1}$.

Neste estudo foram consideradas 3 condições para a superfície do solo: i) Solo nu, sem cobertura; ii) Cobertura morta, com densidade de $2 \mathrm{t} \mathrm{ha}^{-1} \mathrm{e}$ iii) Cobertura morta, com densidade de $4 \mathrm{t} \mathrm{ha}^{-1}$. Para simular a cobertura morta foi utilizada palha de arroz, seco ao ar e uniformemente espalhada sobre o solo garantindo a mesma densidade e percentagem de cobertura da superfície do solo em todo o canal. Para as densidades de cobertura de $2 \mathrm{e} 4 \mathrm{t} \mathrm{ha}^{-1}$ foram obtidas as respectivas percentagens de cobertura de $63,1 \pm 12,3 \%$ e 80,3 $\pm 7,5 \%$. A percentagem de cobertura foi calculada com recurso ao processamento digital de fotografias da vista de topo da superfície do solo coberto (Figuras 1B e 1C).

Para cada condição da superfície do solo foram realizadas 3 repetições do procedimento experimental esquematizado na Figura 2B (i.e. 3 sequências de 5 eventos de precipitação intercalados por períodos secos). No final de cada experimento, o solo e a palha eram removidos e substituídos por solo e palha originais para o experimento seguinte, repetindo as mesmas condições do solo e da palha no início de cada ensaio (e.g. compactação do solo, rugosidade da superfície do solo, teor de água no solo, umidade da palha, distribuição da palha sobre o solo).

O escoamento superficial foi monitorado a jusante do canal de terra através da coleta de amostras sucessivas, a cada $15 \mathrm{~s}$, ao longo da duração dos experimentos. A velocidade do escoamento superficial foi estimada em alguns instantes de cada evento de precipitação utilizando-se um traçador colorido (e.g. corante azul). A velocidade média foi então calculada medindo o tempo de percurso da frente da mancha do traçador e se utilizando um fator de correção experimental igual a 0,67 (Horton et al., 1934; Zhang et al., 2010).

Assumindo o declive longitudinal do canal como sendo uniforme no espaço e no tempo, a altura da lâmina líquida (h) é (Eq. 1):

$$
\mathrm{h}=\frac{\mathrm{Q}_{\mathrm{L}}}{\mathrm{Ub}}
$$

em que:

h - altura da lâmina líquida, $\mathrm{L}$

$\mathrm{Q}_{\mathrm{L}} \quad$ - escoamento a jusante do canal, $\mathrm{L}^{3} \mathrm{~T}^{-1}$

$\mathrm{U}$ - velocidade média do escoamento no canal, $\mathrm{L} \mathrm{T}^{-1}$

b - largura do canal, L

Os regimes dos escoamentos gerados pelos eventos de precipitação foram avaliados através do cálculo do Número de Reynolds (Re) e do Número de Froude (Fr), Eq. 2:

$$
\operatorname{Re}=\frac{\mathrm{Uh}}{\mathrm{v}} ; \mathrm{Fr}=\frac{\mathrm{U}}{\sqrt{\mathrm{gh}}}
$$

em que:

Re - número de Reynolds, adimensional

Fr - número de Froude, adimensional

$\mathrm{v}$ - viscosidade cinemática da água, $\mathrm{L}^{2} \mathrm{~T}^{-1}$

g - aceleração da gravidade, $\mathrm{L} \mathrm{T}^{-2}$ 
A rugosidade da superfície e o atraso do escoamento superficial correspondentes a cada condição de cobertura morta foram avaliados com uso do Coeficiente de Rugosidade de Manning (n) e o Coeficiente de Resistência de DarcyWeisback (f) (Eq. 3):

$$
\mathrm{n}=\frac{\mathrm{h}^{2 / 3} \mathrm{~s}^{1 / 2}}{\mathrm{U}} ; \mathrm{f}=\frac{8 \mathrm{gSh}}{\mathrm{U}^{2}}
$$

em que:

$\mathrm{n}$ - coeficiente de Manning, $\mathrm{T} \mathrm{L}^{-1 / 3}$

f - coeficiente de resistência de Darcy-Weisback, adimensional

S - declive longitudinal do canal, $\mathrm{L} \mathrm{L}^{-1}$

Os resultados dos ensaios laboratoriais foram comparados com os de um modelo numérico, a uma dimensão, com base na teoria da onda cinemática (Singh, 1996; Lima \& Singh, 2002) fazendo uso de simplificações das equações fundamentais da conservação da massa e da quantidade de movimento (Equações de Saint-Venant).

Inicialmente é modelada a precipitação útil que dá origem ao escoamento superficial que serve de entrada à posterior modelagem do escoamento superficial ao longo da direção longitudinal.

A precipitação útil foi estimada em função do espaço e do tempo subtraindo-se as perdas modeladas em função do tempo, a intensidade de precipitação modelada em função do espaço e do tempo. As perdas (e.g. infiltração, interceptação, retenção superficial, evaporação) foram consideradas constantes no espaço e iguais à curva de infiltração do solo.

Assumindo a hipótese da onda cinemática (i.e. perda de carga contínua igual ao declive da superfície, com declive da superfície constante) a equação da onda cinemática pode ser expressa, por unidade de largura, como (Eq. 4):

$$
\frac{\partial \mathrm{h}}{\partial \mathrm{t}}+\frac{\partial \mathrm{q}}{\partial \mathrm{x}}=\mathrm{p}_{\mathrm{u}}
$$

em que:

h - altura da lâmina líquida, $\mathrm{L}$

$\mathrm{q}$ - escoamento, por unidade de largura, $\mathrm{L}^{3} \mathrm{~T}^{-1}$

$\mathrm{p}_{\mathrm{u}}$ - precipitação útil, que dá origem ao escoamento superficial, $\mathrm{L} \mathrm{T}^{-1}$

$\mathrm{t}$ - tempo, $\mathrm{T}$

x - distância ao longo da direção do escoamento, L

Assumindo que o escoamento é apenas função da altura do escoamento (i.e. Relação de Bakemeteff) tem-se, na Eq. 5:

$$
\mathrm{q}=\alpha \mathrm{h}^{\mathrm{m}}
$$

em que:

a - coeficiente de resistência da onda cinemática, adimensional

$\mathrm{m}$ - expoente empírico, adimensional

Considerando regime turbulento, obtém-se uma estimativa para a e m utilizando a fórmula de Manning-Strickler (Eq. 6):

$$
\mathrm{m}=\frac{5}{3} ; \alpha=\frac{\mathrm{S}^{0,5}}{\mathrm{n}}
$$

em que:

$$
\begin{array}{ll}
\mathrm{S} & \text { - declive da superfície, } \mathrm{L} \mathrm{L}^{-1} \\
\mathrm{n} & \text { - coeficiente de Manning, } \mathrm{T} \mathrm{L}^{-1 / 3}
\end{array}
$$

Substituindo a Eq. 5 na Eq. 4, obtém-se a Eq. 7:

$$
\frac{\partial \mathrm{h}}{\partial \mathrm{t}}+\mathrm{m \alpha h}^{\mathrm{m}-1} \frac{\partial \mathrm{h}}{\partial \mathrm{x}}=\mathrm{p}_{\mathrm{u}}
$$

A resolução da Eq. 7 pelo esquema numérico de LaxWendroff, um esquema numérico de $2^{\mathrm{a}}$ ordem, pode ser expresso em forma de diferenças finitas pela Eq. 8 (Lima \& Singh, 2002):

$$
\begin{aligned}
& h_{j}^{i+1}=h_{j}^{i}+\Delta t\left(p_{u_{j}}{ }^{i}-m \alpha \frac{h_{j+1}^{i^{m-1}}+h_{j-1} h_{j-1}^{i m}}{2} \frac{h_{j+1}^{i}-h_{j-1}^{i}}{2 \Delta x}\right)+ \\
& +\frac{(\Delta t)^{2}}{2} \frac{p_{u_{j}}{ }^{i+1}-p_{u_{j}}{ }^{i}}{\Delta t}-m \alpha \frac{(\Delta t)^{2}}{2 \Delta x}\left[\frac{h_{j+1}^{i^{m}-1}+h_{j}^{i^{m-1}}}{2} \times\right. \\
& \times\left(\frac{\mathrm{p}_{\mathrm{u}_{j+1}}{ }^{\mathrm{i}}+\mathrm{p}_{\mathrm{u}_{\mathrm{j}}}{ }^{\mathrm{i}}}{2}-m \alpha \frac{\mathrm{h}_{\mathrm{j}+1}^{\mathrm{i}-1}+\mathrm{h}_{\mathrm{j}}^{\mathrm{i}} \mathrm{i}^{\mathrm{m}-1}}{2} \frac{\mathrm{h}_{\mathrm{j}+1}^{\mathrm{i}}-\mathrm{h}_{\mathrm{j}}^{\mathrm{i}}}{\Delta \mathrm{x}}\right)- \\
& \left.-\frac{\mathrm{h}_{\mathrm{j}}^{\mathrm{i}-1}+\mathrm{h}_{\mathrm{j}-1}^{\mathrm{im}-1}}{2}\left(\frac{\mathrm{p}_{\mathrm{u}_{\mathrm{j}}}{ }^{\mathrm{i}}+\mathrm{p}_{\mathrm{u}_{\mathrm{j}-1}}^{\mathrm{i}}}{2}-\mathrm{m} \alpha \frac{\mathrm{h}_{\mathrm{j}}^{\mathrm{i} \mathrm{m}-1}+\mathrm{h}_{\mathrm{j}-1}^{\mathrm{im}-1}}{2} \frac{\mathrm{h}_{\mathrm{j}}^{\mathrm{i}}-\mathrm{h}_{\mathrm{j}-1}^{\mathrm{i}}}{\Delta \mathrm{x}}\right)\right]
\end{aligned}
$$

em que:

$\begin{array}{ll}\text { j } & \text { - índice que denota a posição }(x) \\ \mathrm{i} & \text { - índice que denota o tempo }(\mathrm{t})\end{array}$

Para a fronteira de jusante da superfície $(\mathrm{x}=\mathrm{L})$ é utilizado um esquema de $1^{\text {a }}$ ordem segundo a Eq. 9 (Lima \& Singh, 2002):

$$
\mathrm{h}_{\mathrm{j}}^{\mathrm{i}+1}=\mathrm{h}_{\mathrm{j}}^{\mathrm{i}}+\Delta \mathrm{t}\left(\mathrm{p}_{\mathrm{u}_{\mathrm{j}}}^{\mathrm{i}}-\mathrm{m \alpha} \frac{\mathrm{h}_{\mathrm{j}}^{\mathrm{i}^{\mathrm{m}-1}}+\mathrm{h}_{\mathrm{j}-1}^{\mathrm{i}^{\mathrm{m}-1}}}{2} \frac{\mathrm{h}_{\mathrm{j}}^{\mathrm{i}}-\mathrm{h}_{\mathrm{j}-1}^{\mathrm{i}}}{\Delta \mathrm{x}}\right)
$$

A solução das Eqs. 8 e 9 requer a verificação das seguintes condições iniciais (Eq. 10) e de fronteira a montante (Eq. 11):

$$
\begin{gathered}
\mathrm{h}(\mathrm{x}, 0)=0, \text { para } 0 \leq \mathrm{x} \leq \mathrm{L} \\
\mathrm{h}(0, \mathrm{t})=0, \text { para } 0 \leq \mathrm{t} \leq \infty
\end{gathered}
$$

em que:

L - comprimento total da superfície ao longo da direção do escoamento, L

Além disto, para garantir a estabilidade numérica do esquema de Lax-Wendroff, deve ser sempre satisfeita a condição de Courant:

$$
\frac{\Delta \mathrm{t}}{\Delta \mathrm{x}}<\frac{1}{\mathrm{mah}^{\mathrm{m}-1}}
$$

No método numérico a precipitação útil que dá origem ao escoamento superficial $\left(\mathrm{p}_{\mathrm{u}}\right)$ foi estimada como sendo o 
resultado da precipitação total (p) subtraindo as perdas (e.g. infiltração, interceptação pela palha, retenção superficial) estimadas a partir de curvas empíricas de infiltração do solo (i). Em função das características da precipitação (e.g. padrão temporal, padrão espacial) e das características da capacidade de infiltração do solo, a precipitação útil $\left(\mathrm{p}_{\mathrm{u}}\right)$ pode variar no espaço (x) e/ou no tempo (t) segundo as Eqs. 13 e 14, a saber:

$$
\begin{gathered}
\mathrm{p}_{\mathrm{u}}(\mathrm{x}, \mathrm{t})=\mathrm{p}(\mathrm{x}, \mathrm{t})-\mathrm{i}(\mathrm{t}), \text { se } \mathrm{p}(\mathrm{x}, \mathrm{t})>\mathrm{i}(\mathrm{t}) \\
\mathrm{p}_{\mathrm{u}}(\mathrm{x}, \mathrm{t})=0, \text { se } \mathrm{p}(\mathrm{x}, \mathrm{t}) \leq \mathrm{i}(\mathrm{t})
\end{gathered}
$$

em que:

$\mathrm{p}_{\mathrm{u}}(\mathrm{x}, \mathrm{t})$ - precipitação útil, $\mathrm{L} \mathrm{T}^{-1}$, que dá origem ao escoamento superficial

$\mathrm{p}(\mathrm{x}, \mathrm{t})$ - precipitação total, $\mathrm{L} \mathrm{T}^{-1}$

$\mathrm{i}(\mathrm{t})$ - infiltração, $\mathrm{L} \mathrm{T}^{-1}$

As curvas empíricas de infiltração (i) foram ajustadas utilizando-se a Equação de Horton (Eq. 15) e consideradas como sendo constantes no espaço $(\mathrm{x})$ variando apenas no tempo $(\mathrm{t})$ :

$$
i(t)=i_{f}+\left(i_{o}-i_{f}\right) e^{-k_{h} t}
$$

em que:

$i(t)$ - infiltração, $\mathrm{L} \mathrm{T}^{-1}$, em função do tempo, $\mathrm{t}$

$\mathrm{i}_{\mathrm{f}} \quad$ - taxa mínima final de infiltração, $\mathrm{L} \mathrm{T}^{-1}$

$i_{o} \quad$ - taxa máxima inicial de infiltração, $\mathrm{L} \mathrm{T}^{-1}$

$\mathrm{k}_{\mathrm{h}}$ - expoente empírico da Equação de Horton, $\mathrm{T}^{-1}$, que representa a constante de decaimento da capacidade de infiltração do solo

Foram adotados os seguintes índices na avaliação de desempenho do modelo numérico: i) Coeficiente de NashSutcliffe, NS, que pode variar entre negativo infinito até 1 (i.e. desempenho perfeito) e ii) Coeficiente de determinação, $\mathrm{R}^{2}$. Considera-se que o desempenho do modelo é aceitável para valores de NS e $\mathrm{R}^{2}$ superiores a 0,75.

\section{Resultados e Discussão}

Nas Tabelas 2 e 3 se encontram expressos os diferentes parâmetros calculados para avaliar as características hidráulicas, os regimes de escoamento e as características da superfície observados nos vários ensaios laboratoriais.

A cobertura morta originou não apenas diminuição significativa do escoamento a jusante do canal, da velocidade do escoamento superficial no canal mas também aumento da altura da lâmina líquida cujos resultados estão de acordo com outros estudos (Chen et al., 2007; Santos et al., 2010). Em média verificou-se, para as cinco chuvas, diminuição de 10 e $39 \%$ no escoamento de ponta e de 44 e $53 \%$ na velocidade do escoamento superficial, respectivamente para as densidades de cobertura de 2 e $4 \mathrm{t} \mathrm{ha}^{-1}$. Para a condição de densidade de cobertura de $4 \mathrm{t} \mathrm{ha}^{-1}$, em uma das repetições, chegou-se a verificar ausência de escoamento a jusante do canal durante o $1^{\circ}$ evento de precipitação. Deste modo não foi possível estimar a velocidade do escoamento superficial durante o $1^{\circ}$ evento de precipitação na condição de maior densidade de cobertura morta. Em relação à altura da lâmina líquida não se verificaram diferenças significativas entre os resultados obtidos para as duas situações com cobertura morta sendo que em alguns casos a altura da lâmina líquida chegou a ser superior para a situação com densidade de cobertura de $2 \mathrm{t} \mathrm{ha}^{-1}$.

O número de Reynolds e o número de Froude diminuíram com a aplicação de cobertura morta. Para todas as condições da superfície do solo o número de Reynolds aumentou com a intensidade de precipitação embora não se tenha verificado uma relação clara entre o número de Froude e a intensidade de precipitação. A cobertura morta originou aumento do Coeficiente de Rugosidade de Manning e do Coeficiente de

\begin{tabular}{|c|c|c|c|c|c|c|c|c|c|c|}
\hline \multirow{3}{*}{$\begin{array}{l}\text { Ordem do evento } \\
\text { de precipitação }\end{array}$} & \multirow{3}{*}{$\begin{array}{l}\text { Intensidade de } \\
\text { precipitação } \\
\left(\mathrm{mm} \mathrm{h} \mathbf{h}^{-1}\right)\end{array}$} & \multicolumn{9}{|c|}{ Densidade de cobertura (t ha ${ }^{1}$ ) } \\
\hline & & 0 & 2 & 4 & 0 & 2 & 4 & 0 & 2 & 4 \\
\hline & & \multicolumn{3}{|c|}{$Q_{p}\left(\mathrm{ml} \mathrm{s}^{-1}\right)$} & \multicolumn{3}{|c|}{$\mathrm{U}\left(\mathrm{m} \mathrm{s}^{-1}\right)$} & \multicolumn{3}{|c|}{$\mathrm{h}(\mathrm{mm})$} \\
\hline 1 & 57 & 10,83 & 7,71 & 2,71 & 0,041 & 0,023 & - & 0,891 & 1,072 & - \\
\hline 2 & 84 & 19,77 & 18,00 & 13,06 & 0,045 & 0,033 & 0,023 & 1,482 & 1,856 & 1,919 \\
\hline 3 & 112 & 27,01 & 24,51 & 20,04 & 0,057 & 0,033 & 0,026 & 1,597 & 2,482 & 2,565 \\
\hline \multirow{2}{*}{4} & 112 & 26,65 & 25,87 & 21,06 & 0,061 & 0,034 & 0,030 & 1,466 & 2,561 & 2,358 \\
\hline & 57 & 13,62 & 12,64 & 8,38 & 0,049 & 0,024 & 0,023 & 0,931 & 1,768 & 1,234 \\
\hline 5 & 112 & 27,55 & 25,08 & 22,04 & 0,066 & 0,032 & 0,028 & 1,389 & 2,604 & 2,656 \\
\hline
\end{tabular}

Tabela 2. Escoamento de ponta $\left(Q_{p}\right)$, velocidade média do escoamento superficial (U) e altura da lâmina líquida (h), observadas nos experimentos para as três condições da superfície do solo e para os cinco eventos de precipitação

Tabela 3. Características hidráulicas do escoamento superficial observado nos experimentos para as três condições

\begin{tabular}{|c|c|c|c|c|c|c|c|c|c|c|c|c|c|}
\hline \multirow{3}{*}{$\begin{array}{c}\text { Ordem do } \\
\text { evento de } \\
\text { precipitação }\end{array}$} & \multirow{3}{*}{$\begin{array}{l}\text { Intensidade de } \\
\text { precipitação } \\
\left(\mathrm{mm} \mathrm{h}^{-1}\right)\end{array}$} & \multicolumn{12}{|c|}{ Densidade de cobertura (t ha') } \\
\hline & & 0 & 2 & 4 & 0 & 2 & 4 & 0 & 2 & 4 & 0 & 2 & 4 \\
\hline & & & $\operatorname{Re}(-)$ & & \multicolumn{3}{|c|}{$\operatorname{Fr}(-)$} & \multicolumn{3}{|c|}{$n\left(s^{-1 / 3}\right)$} & \multicolumn{3}{|c|}{$f(-)$} \\
\hline 1 & 57 & 30,82 & 21,92 & 7,70 & 0,436 & 0,226 & 0,238 & 0,073 & 0,144 & 0,121 & 4,38 & 16,28 & 14,16 \\
\hline 2 & 84 & 56,24 & 51,20 & 37,14 & 0,373 & 0,248 & 0,171 & 0,093 & 0,151 & 0,224 & 5,95 & 15,13 & 33,54 \\
\hline 3 & 112 & 76,83 & 69,73 & 57,02 & 0,455 & 0,216 & 0,166 & 0,077 & 0,178 & 0,231 & 3,98 & 18,89 & 31,34 \\
\hline \multirow[b]{2}{*}{4} & 112 & 75,83 & 73,60 & 59,90 & 0,507 & 0,214 & 0,196 & 0,067 & 0,176 & 0,189 & 3,14 & 18,00 & 21,28 \\
\hline & 57 & 38,74 & 35,96 & 23,85 & 0,512 & 0,181 & 0,207 & 0,062 & 0,197 & 0,161 & 3,08 & 25,46 & 19,18 \\
\hline \multirow{2}{*}{5} & 57 & 37,74 & 35,05 & 24,96 & 0,570 & 0,193 & 0,214 & 0,055 & 0,180 & 0,157 & 2,47 & 21,58 & 18,18 \\
\hline & 112 & 78,37 & 71,35 & 62,70 & 0,573 & 0,201 & 0,171 & 0,060 & 0,187 & 0,220 & 2,53 & 19,99 & 27,32 \\
\hline
\end{tabular}
da superfície do solo e para os cinco eventos de precipitação: Número de Reynolds (Re), Número de Froude (Fr), Coeficiente de Rugosidade de Manning (n) e Coeficiente de Resistência de Darcy-Weisback (f) 
Resistência de Darcy-Weisback; apesar disto, nenhum desses coeficientes apresentou uma relação clara com a intensidade de precipitação. Em média, para os cinco eventos de precipitação o Coeficiente de Rugosidade de Manning variou entre 0,070 $\mathrm{s} \mathrm{m}^{-1 / 3}$, para o solo nu e 0,173 e $0,186 \mathrm{~s} \mathrm{~m}^{-1 / 3}$, respectivamente, para as densidades de cobertura morta de 2 e $4 \mathrm{t} \mathrm{ha}^{-1}$, representando um aumento de 149 e $168 \%$, respectivamente. De forma similar foram observados, em Pan \& Shangguan (2006) diminuição do número de Reynolds e do número de Froude e aumento do Coeficiente de Rugosidade de Manning e do Coeficiente de Resistência de Darcy-Weisback com o aumento da cobertura do solo com grama.

Na Figura 3 são apresentadas as curvas empíricas de infiltração utilizadas na simulação numérica, ajustadas pela Equação de Horton (Eq. 15) segundo os parâmetros empíricos presentes na Tabela 4. Para cada condição da superfície do solo (i.e. solo nu, densidade de cobertura de 2 e $4 \mathrm{t} \mathrm{ha}^{-1}$ ) foram consideradas duas curvas de infiltração: i) Uma curva de infiltração para a condição inicial de solo seco, que foi aplicada na simulação numérica do $1^{\circ}$ evento de precipitação e ii) Uma curva de infiltração para a condição inicial de solo úmido, utilizada na simulação dos $2^{\circ}, 3^{\circ}, 4^{\circ}$ e $5^{\circ}$ eventos de precipitação.

Apresentam-se, na Figura 4, os hidrogramas de escoamento superficial observados em laboratório e simulados numericamente. $\mathrm{O}$ ajuste dos hidrogramas simulados numericamente aos observados em laboratório, foi adequado, verificando o bom ajuste observado em Deng et al. (2008) para solo nu; para o $1^{\circ}$ evento de precipitação, com condição inicial de solo seco, as diferenças entre os valores simulados e os valores observados foram superiores principalmente na situação de maior densidade de cobertura morta. À medida que as umidades iniciais do solo aumentaram, o desempenho do modelo numérico foi superior.

Na Figura 5 é apresentada a comparação entre o escoamento superficial simulado numericamente e o observado em

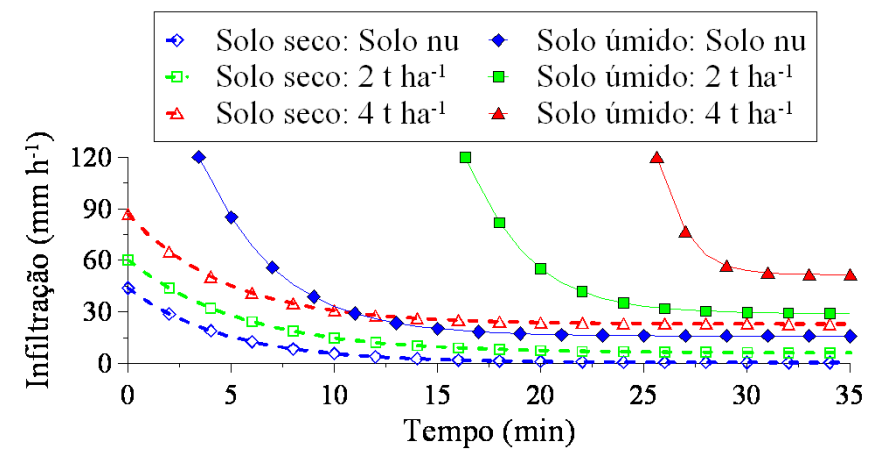

Figura 3. Curvas empíricas de infiltração obtidas dos experimentos para condições iniciais de solo seco $\left(1^{\circ}\right.$ evento de precipitação) e condições iniciais de solo úmido $\left(2^{\circ}, 3^{\circ}, 4^{\circ}\right.$ e $5^{\circ}$ eventos de precipitação) laboratório. Em geral, os valores simulados numericamente foram ligeiramente superestimados enquanto as diferenças

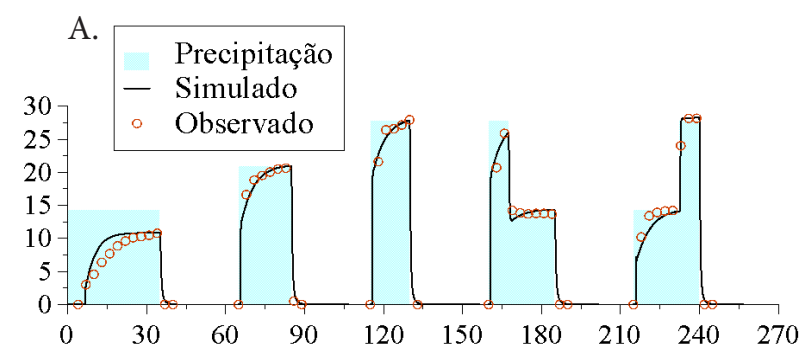

B.

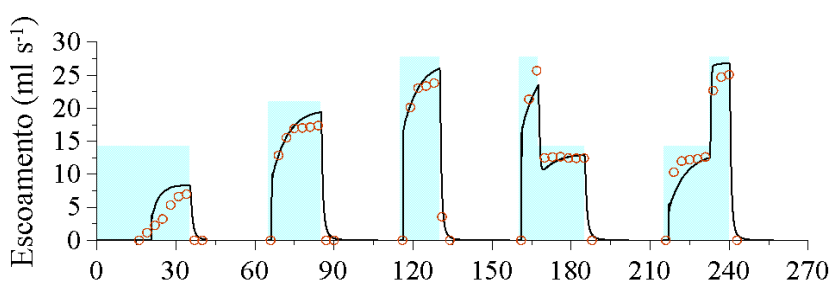

C.

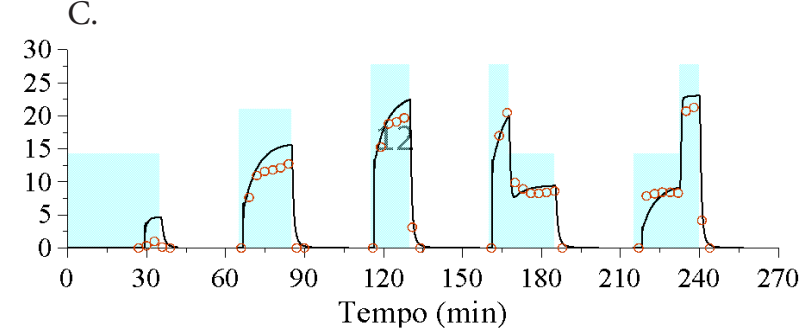

Figura 4. Comparação entre os hidrogramas de escoamento superficial observados nos experimentos e simulados numericamente: Solo nu (A), Densidade de cobertura de $2 \mathrm{t} \mathrm{ha}^{-1}(\mathrm{~B})$, Densidade de cobertura de $4 \mathrm{t} \mathrm{ha}^{-1}(\mathrm{C})$

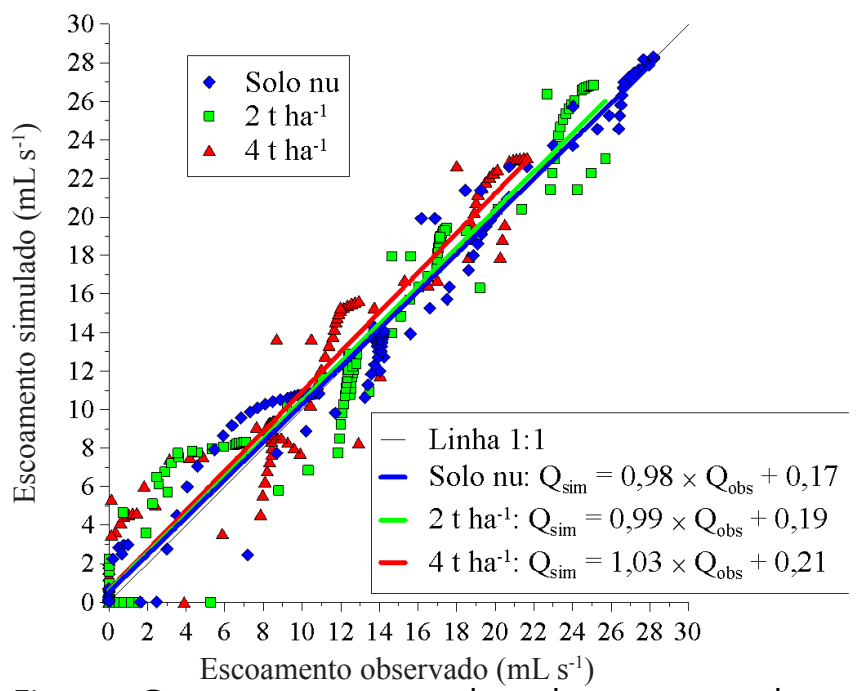

Figura 5. Comparação entre os valores de escoamento observados nos experimentos $\left(\mathrm{Q}_{\mathrm{obs}}\right)$ e simulados numericamente $\left(\mathrm{Q}_{\text {sim }}\right)$

Tabela 4. Parâmetros empíricos da Equação de Horton obtidos dos experimentos para condições iniciais de solo seco ( $1^{\circ}$ evento de precipitação) e condições iniciais de solo úmido $\left(2^{\circ}, 3^{\circ}, 4^{\circ}\right.$ e $5^{\circ}$ eventos de precipitação): taxa máxima inicial de infiltração $\left(i_{o}\right)$, taxa mínima final de infiltração $\left(i_{f}\right)$ e constante de decaimento da capacidade de infiltração do solo $\left(k_{h}\right)$

\begin{tabular}{|c|c|c|c|c|c|c|c|c|c|}
\hline \multirow{3}{*}{$\begin{array}{c}\text { Condição } \\
\text { inicial do solo }\end{array}$} & \multicolumn{9}{|c|}{ Densidade de cobertura ( $\left.\mathrm{t} \mathrm{ha}{ }^{1}\right)$} \\
\hline & 0 & 2 & 4 & 0 & 2 & 4 & 0 & 2 & 4 \\
\hline & & $i_{0}\left(m m ~ h^{-1}\right)$ & & & $(\mathrm{mm} \mathrm{h}$ & & & $k_{h}\left(h^{-1}\right)$ & \\
\hline Solo seco & 292,61 & 32822,75 & $5,80 \times 10^{10}$ & 15,95 & 28,94 & 51,62 & 16,63 & 21,42 & 47,88 \\
\hline Solo úmido & 43,81 & 60,12 & 87,01 & 0,36 & 5,98 & 22,97 & 12,67 & 10,80 & 12,67 \\
\hline
\end{tabular}


entre os resultados numéricos e os experimentais aumentaram com a densidade de cobertura morta.

$\mathrm{Na}$ Tabela 5 se encontram os índices de avaliação de desempenho do modelo numérico. Em geral, o modelo da onda cinemática a uma dimensão, conseguiu simular o escoamento superficial com um ajuste bastante elevado. O desempenho do modelo aumentou com a intensidade média de precipitação e diminuiu com a densidade de cobertura morta; apenas para a situação de maior densidade de cobertura $\left(4 \mathrm{t} \mathrm{ha}^{-1}\right) \mathrm{e}$ condição inicial de solo seco ( $1^{\circ}$ evento de precipitação) é que o desempenho do modelo não foi considerado aceitável, com valor do coeficiente de Nash-Sutcliffe abaixo de 0,75.

Tabela 5. Índices de avaliação de desempenho do modelo numérico usado para simular o escoamento superficial, para as três condições da superfície do solo e cinco chuvas simuladas em laboratório

\begin{tabular}{|c|c|c|c|c|c|c|}
\hline \multirow{3}{*}{$\begin{array}{c}\text { Ordem } \\
\text { do evento } \\
\text { de precipitação }\end{array}$} & \multicolumn{6}{|c|}{ Densidade de cobertura ( $\mathrm{th} \mathrm{a}^{1}$ ) } \\
\hline & $\mathbf{0}$ & 2 & 4 & 0 & 2 & 4 \\
\hline & \multicolumn{3}{|c|}{ NS } & \multicolumn{3}{|c|}{$\mathbf{r}^{2}$} \\
\hline 1 & 0,906 & 0,772 & 0,291 & 0,941 & 0,875 & 0,778 \\
\hline 2 & 0,988 & 0,963 & 0,839 & 0,989 & 0,981 & 0,950 \\
\hline 3 & 0,991 & 0,974 & 0,942 & 0,992 & 0,985 & 0,975 \\
\hline 4 & 0,976 & 0,956 & 0,924 & 0,978 & 0,969 & 0,938 \\
\hline 5 & 0,980 & 0,937 & 0,946 & 0,983 & 0,942 & 0,953 \\
\hline
\end{tabular}

\section{Conclusões}

1. O modelo da onda cinemática permitiu bom ajuste dos valores simulados aos valores observados em laboratório, sobretudo para situações de solo nu.

2. A cobertura morta influenciou a modelagem do escoamento superficial diminuindo o ajuste dos valores simulados aos valores observados; ainda assim, o desempenho do modelo pode ser considerado aceitável.

3. Em geral, os valores do coeficiente de Nash-Sutcliffe foram superiores a 0,75 e valores do coeficiente de determinação superiores a 0,90 .

\section{Agradecimentos}

Os autores agradecem o financiamento concedido pela Fundação para a Ciência e a Tecnologia (FCT), Portugal, no âmbito do projeto de investigação PTDC/ECM/105446/2008 e ao suporte financeiro do CNPq, FINEP e CAPES.

\section{Literatura Citada}

Cerdà, A.; Ibáñez, S.; Calvo, A. Design and operation of a small and portable rainfall simulator for rugged terrain. Soil Technology, v.11, p.163-170, 1997. http://dx.doi.org/10.1016/S09333630(96)00135-3

Chen, L.; Huang, Z.; Gong, J.; Fu, B.; Huang, Y. The effect of land cover/vegetation on soil water dynamic in the hilly area of the loess plateau, China. Catena, v.70, p.200-208, 2007. http://dx.doi. org/10.1016/j.catena.2006.08.007

Christiansen, J. E. Irrigation by sprinkling. Berkeley: University of California, 1942.124p. California Agricultural Experiment Station Bulletin 670 .

Deng, Z. Q.; Lima, J. L. M. P. de; Jung, H.S. Sediment transport ratebased model for rainfall-induced soil erosion. Catena, v.76, p.5462, 2008. http://dx.doi.org/10.1016/j.catena.2008.09.005
Giesen, N. van de; Stomph, T. J.; Ajayi, A. E.; Bagayoko, F. Scale effects in Hortonian surface runoff on agricultural slopes in West Africa: Field data and models. Agriculture, Ecosystems and Environment, v.142, p.95-101, 2011. http://dx.doi.org/10.1016/j.agee.2010.06.006

Horton R. E.; Leach, H. R.; Vliet, R. van. Laminar sheet-flow. Transactions of the American Geophysical Union, v.15, p.393-404, 1934. http://dx.doi.org/10.1029/TR015i002p00393

Lima, J. L. M. P. de; Singh, V. P. The influence of the pattern of moving rainstorms on overland flow. Advances in Water Resources, v.25, p.817-828, 2002. http://dx.doi.org/10.1016/S0309-1708(02)00067-2

Lima, J. L. M. P. de; Singh, V. P.; Lima, M. I. P. de. The influence of storm movement on water erosion: Storm direction and velocity effects. Catena, v.52, p.39-56, 2003. http://dx.doi.org/10.1016/ S0341-8162(02)00149-2

Lima, J. L. M. P. de; Torfs, P. J. J. F.; Singh, V. P. A mathematical model for evaluating the effect of wind on downward-spraying rainfall simulators. Catena, v.46, p.221-241, 2002. http://dx.doi. org/10.1016/S0341-8162(01)00171-0

Molen, W. H. van de; Torfs, P. J. J. F.; Lima, J. L. M. P. de. Water depths at the upper boundary for overland flow on small gradients. Journal of Hydrology, v.171, p.93-102, 1995. http://dx.doi. org/10.1016/0022-1694(95)02718-5

Montenegro, A. A. A.; Abrantes, J. R. C. B.; Lima, J. L. M. P. de; Singh, V. P.; Santos T. E. M. Impact of mulching on soil and water dynamics under intermittent simulated rainfall. Catena, v.109, p.139-149, 2013. http://dx.doi.org/10.1016/j.catena.2013.03.018

Pan, C.; Shangguan, Z. Runoff hydraulic characteristics and sediment generation in sloped grassplots under simulated rainfall conditions. Journal of Hydrology, v.331, p.178-185, 2006. http:// dx.doi.org/10.1016/j.jhydrol.2006.05.011

Pérez-Latorre, F. J.; Castro, L. de; Delgado, A. A comparison of two variable intensity rainfall simulators for runoff studies. Soil \& Tillage Research, v.107, p.11-16, 2010. http://dx.doi.org/10.1016/j. still.2009.12.009

Ran, Q.; Su, D.; Li, P.; He, Z. Experimental study of the impact of rainfall characteristics on runoff generation and soil erosion. Journal of Hydrology, v.424-425, p.99-111, 2012. http://dx.doi. org/10.1016/j.jhydrol.2011.12.035

Römkens, M. J. M.; Helming, K.; Prasad, S. N. Soil erosion under different rainfall intensities, surface roughness, and soil water regimes.Catena, v.46, p.103-123, 2001. http://dx.doi.org/10.1016/ S0341-8162(01)00161-8

Santos, T. E. M.; Montenegro, A. A. A.; Silva D. D. Umidade do solo no semiárido pernambucano usando-se reflectometria no domínio do tempo (TDR). Revista Brasileira de Engenharia Agrícola e Ambiental, v.15, p.670-679. 2011. http://dx.doi.org/10.1590/ S1415-43662011000700004

Santos, T. E. M.; Silva, D. D.; Montenegro, A. A. A. Temporal variability of soil water content under different surface conditions in the semiarid region of the Pernambuco state. Revista Brasileira de Ciências do Solo, v.34, p.1733-1741, 2010. http://dx.doi. org/10.1590/S0100-06832010000500025

Shen, J. Y.; Zhao, D. D.; Han, H. F.; Zhou, X. B.; Li, Q. Q. Effects of straw mulching on water consumption characteristics and yield of different types of summer maize plants. Plant, Soil and Environment, v.58, p.161-166, 2012.

Singh, V. P. Kinematic wave modelling in water resources: Surface-water hydrology. Chichester: John Wiley and Sons Ltd, 1996. 1424p.

Zhang, G. H.; Luo, R. T.; Cao, Y.; Shen, R. C.; Zhang, X. C. Correction factor to dye-measured flow velocity under varying water and sediment discharges. Journal of Hydrology, v.389, p.205-213, 2010. http://dx.doi.org/10.1016/j.jhydrol.2010.05.050 